Revista de Psicología de la PUCP. Vol. XII. No 2. 1994

\title{
DEPRESION Y AUTOCONCEPTO EN NIÑOS INSTITUCIONALIZADOS Y NO INSTITUCIONALIZADOS
}

\author{
Luis. F. Raffo Benavides ${ }^{1}$
}

El estudio investiga la depresión y el autoconcepto en grupos de niños Peruanos expuestos a situaciones socioafectivas negativas, destacando la importancia y características específicas de este desorden en el campo psicológico y clínico. Fueron seleccionados 35 niños institucionalizados, 30 niños de zona marginal de Lima y 20 niños con diagnóstico de depresión; todos varones entre 8 y 14 años. Se estudió la correlación entre ambas variables, se comparó los puntajes a nivel intergrupal y se adaptó el CDI (Children Depressive Inventory, Kovacs, 1983). Se obtuvo una correlación negativa y significativa entre las variables de depresión y autoconcepto. Asimismo, se encuentra que la institucionalización y el bajo nivel socioeconómico tienen influencia en el grado de depresión hallado en los niños examinados, en cambio tienen poca trascendencia en el nivel de autoconcepto. La evaluación de la validez y confiabillidad de los instrumentos utilizados brinda resultados óptimos, presentándose datos adicionales importantes.

Palabras claves: Depresión, autoconcepto, niño institucionalizado - CDI (Children's Depression Inventory), diagnóstico.

Depression and self-Concept in institutionalized and non-instituionalized children This article reports a research on depression and self-concept in Peruvian children exposed to negarive socioafective situations. It emphasizes the importance and specific characteristics of depression both from a psychological and dinical point of view. The sample consisted of boys between 8 and 14 years old, 35 institutionalized, 30 from a marginal district of Lima and 20 with a depression diagnosis. The objective of the study was to analyse the correlation between both variables, compare the scores in the three groups, and the adaptation of the CDI (Children Depressive Inventory, Kovacs, 1983). Results show a signifcative negative correlation between depression and self-concept. It is found that institutionalization and low SES influence the degree of depression, however, it has less importance in self-concept. The instruments are valid and reliable.

Key words: Depression, self-concept - institutionalized child - CDI (Children's Depression Inventory), diagnosis

1 Psicólogo, Docente de la Pontificia Universidad Católica del Perú. Egresado de esta universidad, ha realizado estudios de Maestría en Psicología Educativa en la U.N.M.S.M. Realiza investigación en el campo de la Psicología de Niños y Adolescentes. 



\section{Introducción}

Admitiendo que el término depresión no tiene el "mismo significado" para el nińo y el adulto, su naturaleza es compleja. Es importante distinguir tal como bien señala Ajurriaguerra (1973), el afecto de la enfermedad depresiva, el momento de la forma de ser depresiva y las fases de los procesos depresivos. Específicamente la depresión en el niño es una entidad propia (Tesiny, 1982) y clínicamente aceptada (Malmquist, 1983).

Dentro de las diversas definiciones y clasificaciones de síntomas en base a criterios clínicos (DSM III, 1980; Carey y otros, 1987) es posible precisar una definición más funcional que caracteriza la depresión en el niño como un estado manifestado por la reducción tanto del entusiasmo como de la capacidad de experimentar placer (Lefkowitz y Burton, 1978). Los autores señalan cuatro áreas de funcionamiento que pueden estar afectadas:

(a) Afectiva, por manifestaciones de disforia.

(b) Cognitiva, por ideas y manifestaciones de desprecio hacia sí mismo.

(c) Motivacional, por un desempeño decreciente y desinterés.

(d) Vegetativa, por fatiga, problemas en el dormir y bajo apetito.

Investigaciones sobre el tema sostienen que el niño deprimido al autoevaluarse puede manifestar por sí mismo los indicadores negativos mencionados (Kovacs, 1983). Ello se explica por las graves distorsiones en el desarrollo afectivo, cognitivo y social (Bell-Dolan et al., 1993) y fundamentalmente por la presencia de un bajo nivel e inadecuado desarrollo del autoconcepto, que representa el primer indicador del estado depresivo en el niño y generador de errores cognitivos (Stevenson y Rommey, 1984; Leitenberg, 1986). 
En líneas generales se admite, además, que la depresión en el niño está relacionada a procesos y experiencias deficitarias provenientes de su medio ambiente externo y, principalmente, de las personas significativas de su entorno familiar frustrante, marginal, desestructurado y desorganizado (Alarcón, 1986; Green, 1981; Puyuelo, 1984) y de estructura compleja (Frias y otros, 1992). Se reconoce también la prevalencia de depresión en niños de 8 a 13 años con bajo rendimiento académico y dificultades de aprendizaje, aspectos que son característicos en niños de familia marginal (Bleschman et al., 1986; Stevenson y Romney, 1984).

En relación al niño institucionalizado, podemos seńalar que proviene generalmente de un medio social marginal, siendo notorios una serie de déficits y alteraciones en su desarrollo afectivo y adaptación personal, entre ellos depresión y un pobre nivel de autoconcepto.

Diversos estudios en el campo clínico sobre la depresión en niños destacan una serie de características específicas que presenta este desorden. $\mathrm{Al}$ respecto, investigaciones reportan una correlación negativa entre el nivel de depresión y el nivel de autoconcepto en el nińo (Lefkowitz y Tesiny, 1980; Leitenberg et al.,1986; Tems y otros 1993).

Pensamos que la relación entre estas variables también se presenta en el niño institucionalizado. Las vivencias anteriores a su internamiento dentro de un medio sociocultural y familiar marginal y deficitario, como la percepción de su ingreso y estadía en la institución, generan en este tipo de nińos sentimientos de abandono, rechazo y desamparo personal. Esto estructura en ellos una experiencia depresiva, un pobre concepto de sí mismo con consecuencias negativas en el plano emocional, cognitivo y social (Kovacs, 1983).

La presente investigación responde fundamentalmente a las siguientes interrogantes:

1. ¿Cuál es el grado de depresión y nivel de autoconcepto que presentan los nińos institucionalizados?

2. ¿Presentan los niños institucionalizados indicadores de depresión y autoconcepto similares a los encontrados en niños con diagnóstico de depresión y que reciben atención psiquiátrica? 
3. ¿Qué indicadores de las variables estudiadas presentan los niños pobres de zonas marginales?

\section{Metodología}

\section{Muestra}

Todos los nińos seleccionados fueron varones entre 8 y 13 años con nivel de escolaridad de 3er. a 6to. grado y un C.I. normal promedio de acuerdo a la Escala de Inteligencia para Niños de Wechsler-revisada. Se trabajó con una muestra intencional, no probabilística, tomándose en cuenta indicadores socioeconómicos para la selección de los niños. La muestra estuvo conformada por tres grupos estáticos de comparación: 30 nińos institucionalizados (Grupo 1), 20 niños con diagnóstico de depresión y atención psiquiátrica (Grupo 2) y 35 niños de un centro educativo de zona marginal de Lima Metropolitana (Grupo 3).

Para la selección se tomó en cuenta información previa de cada niño (historia personal, C.I, grado escolar, nivel socioeconómico). En el grupo de niños con atención psiquiátrica se obtuvo información en relación al diagnóstico de depresión según criterios del DSM III, DSM III-R y el ICE9-CM.

\section{Instrumentos}

Para evaluar la depresión se utilizó el Inventario de Depresión para niños de Kovacs (1983) (CDI), instrumento clínico en base a autoreportes de síntomas depresivos en el niño. Consta de 27 ítemes que cuantifica un rango de los mismos a nivel del humor, capacidad de experimentar placer, funciones vegetativas, autoconcepto y conducta interpersonal, teniendo en cuenta así mismo diferentes contextos importantes para el niño (p.e medio escolar). Es aplicable a niños entre 8 y 13 años, requiriendo adecuada capacidad de lectura. Cada ítem contiene 3 oraciones que describen manifestaciones depresivas, dentro de un rango que está valorizado en cada item de 0 a 2 según la severidad del síntoma. El niño elige una de ellas, siendo el rango potencial de puntaje total entre 0 a 54 . Un puntaje superior a 11 determina la presencia de signos depresivos (Kovacs, 1983; Hodges, 
1983). Se administra en pequeńos grupos o individualmente si así lo requiere el estado psicológico del niño.

Su confiabilidad reporta altos coeficientes de consistencia interna tanto para la muestra clínica $(0.86)$ como para la muestra escolar (0.87). La correlación ítem-puntaje total reporta un rango general entre 0.25-0.62, siendo el coeficiente test-retest alto (0.84) con un intervalo de 9 semanas (Kovacs, 1983). La validez del inventario original fue de tipo concurrente en relación a dos variables; ansiedad manifiesta $(r=0.65, p<0.01)$ y autoestima $(r=-0.59, \mathrm{p}<0.01)$.

Inicialmente, se evaluó la validez de contenido del CDI. Tres psicólogos expertos y con dominio en el idioma inglés realizaron la doble traducción del inventario. El CDI traducido fue sometido a criterio de jueces, contando con una definición operacional de depresión en niños. Ellos brindaron su opinión sobre los ítemes con resultado satisfactorio (todos con más de $\mathbf{8 0} \%$ de aceptación).

Para la evaluación del autoconcepto se utilizó la escala de Piers-Harris, adaptada en el Perú por Rueda (1987) en muestras de niños institucionalizados y no institucionalizados. La escala consta de 80 ítemes que deben ser respondidos por el niño con sí o no. Estudia esta variable en 6 áreas (Conducta General y Conducta Social, Estatus Intelectual, Apariencia, Popularidad, Satisfacción). Es aplicable a niños de ambos sexos entre 8 y 13 ańos, proporcionando datos a nivel general y en cada uno de los factores señalados, según la dirección establecida e interpretados de la siguiente manera: cuanto más alto el puntaje, más positivo es el atributo. Un puntaje mayor a 61 indica un autoconcepto alto entre 46 y 60 , nivel promedio y un puntaje por debajo de 46 indica un nivel bajo (Piers-Harris, 1967). Investigaciones hechas en el Perú, reportan altos índices de validez y confiabilidad de diverso tipo realizada con niños procedentes de medios institucionalizados y de zona marginal (Avalos, 1979; Rueda, 1987).

\section{Procedimiento}

La aplicación de los instrumentos fue individual en cada uno de los grupos y siguiendo de manera precisa las instrucciones recomendadas por los autores de los respectivos inventarios. 
Para el tratamiento estadístico de los datos, se utilizó el método de Kruskall-Wallis, prueba no paramétrica de análisis de varianza simple, a fin de examinar la existencia de diferencias significativas en general entre los grupos para cada una de las dos variables. Como condición previa a esta prueba se utilizó el estadístico de Kolmogorov-Smirnov (Prueba de Bondad de Ajuste), comprobándose que las variables examinadas tienen una distribución normal y continua. Seguidamente se procedió a examinar las diferencias específicas entre los grupos a través de la prueba $T$, técnica paramétrica empleada para analizar datos de muestras independientes (Grupos) de poblaciones con distribución normal, y obtenidas por escalas de intervalo.

\section{Resultados y Discusión}

En relación a las propiedades psicométricas del CDI, evaluadas para su adaptación en nuestro medio los resultados son favorables. A parte de la validez de contenido, se estudió la validez concurrente del CDI en base a su correlación con la escala de Piers-Harris, obteniéndose un coeficiente significativo $(r=-0.48, p<0.01, n=85)$, lo que indica que a mayor severidad de desorden depresivo en el niño se encuentra un menor nivel de autoconcepto. Así mismo la validez de construcción del inventario, se estudió a partir del Análisis Factorial de los componentes fundamentales, utilizando el procedimiento "Varimax Rotation" (Hetsel y Matson, 1984) que proporcionaron inicialmente 10 factores seleccionándose sólo cuatro de ellos por contener cada uno más de cuatro ítemes, número considerado apropiado para otorgar consistencia a los siguientes componentes derivados del CDI: Afectividad, Imagen-ideación, Relaciones Interpersonales y Culpairritabilidad.

En relación a la confiabilidad, se obtuvo un coeficiente de homogeneidad de Kuder-Richardson, Fórmula 20 (K-R20) de $\mathrm{r}=0.81$ para la muestra total que equivale a un nivel alto de consistencia interna. El CDI presenta una aceptable consistencia interna en el grupo psiquiátrico $(r=0.79)$, pero mayor en los otros dos grupos, institucionalizado $(r=0.85)$ y no institucionalizado de zona marginal $(r=0.83)$. A nivel de los ítemes se estudió la confiabilidad del CDI en base a correlaciones ítem/puntaje total, siendo todos los coeficientes hallados significativos dentro de un rango de $r=0.21$ 
a $\mathrm{r}=0.63(\mathrm{p}<0.05, \mathrm{n}=85)$. Evidentemente las medidas de depresión obtenidas a través de los ítemes son consistentes para explicar de manera válida un patrón específico de respuestas relacionados a la experiencia depresiva en los niños examinados.

El análisis de varianza reporta la existencia de diferencias a nivel general entre los grupos en indicadores depresivos ( $\mathrm{kw}=6.34, \mathrm{~F}=3.23, \mathrm{gl}=2$, $\mathrm{p}=0.042$ ). Resultados más específicos a nivel comparativo entre los grupos examinados (Prueba T) indican: a) La ausencia de diferencias significativas en el nivel de depresión entre el grupo de niños institucionalizados y los niños de zona marginal; b) Diferencias significativas en relación a indicadores de depresión entre el grupo de niños institucionalizados y el grupo de niños con atención psiquiátrica; c) La ausencia de diferencias significativas entre los dos grupos no institucionalizados (marginal y clínico), que presentan similares características de depresión. Los resultados se presentan en el Cuadro 1.

Cuadro 1: Prueba T - Intergrupo - Puntajes CDI

\begin{tabular}{|lcrrrr|}
\hline \multicolumn{1}{|c}{ GRUPO } & $\mathrm{N}$ & $\overline{\mathrm{X}}$ & $\mathrm{DS}$ & $\mathrm{T}-$ test \\
\hline $\begin{array}{l}\text { 1. Niños } \\
\text { Institucionalizados }\end{array}$ & 35 & 11.63 & 4.766 & $\mathrm{~T}=2.48$ & $\mathrm{G} 1=\mathrm{G} 2$ \\
$\begin{array}{l}\text { 2. Niños de Zona } \\
\text { Marginal }\end{array}$ & 30 & 14.07 & 7.817 & & \\
\hline $\begin{array}{l}\text { 1. Niños } \\
\text { Institucionalizados }\end{array}$ & 35 & 11.63 & 4.766 & $\mathrm{~T}=2.48 *$ & $\mathrm{G} 1<\mathrm{G} 2$ \\
$\begin{array}{l}\text { 2. Niños con } \\
\text { Atención } \\
\text { Psiquiátrica }\end{array}$ & 20 & 16.1 & 6.688 & & \\
\hline $\begin{array}{l}\text { 1. Nińos de Zona } \\
\text { Marginal }\end{array}$ & 30 & 14.07 & 7.817 & $\mathrm{~T}=2.48$ & $\mathrm{G} 1=\mathrm{G} 2$ \\
$\begin{array}{l}\text { 2. Nińos con } \\
\text { Atención } \\
\text { Psiquiátrica }\end{array}$ & 20 & 16.1 & 6.688 & & \\
\hline
\end{tabular}

${ }^{*} \mathrm{p}<.05$. 
Si bien es cierto, todos los grupos presentan depresión a través del CDI, sorprende el puntaje promedio obtenido por el grupo de niños de zona marginal que se ubica en un nivel intermedio entre un desorden leve (grupo institucionalizado) y un nivel de depresión mayor (grupo clínico). La influencia negativa que ejercen las características deficitarias del ambiente sociocultural y familiar sobre el desarrollo del niño, notoriamente crítico en esta población, parecen determinar el alto nivel de depresión encontrado en los niños escolares procedentes de esta población.

Cuadro 2: Prueba T - Puntajes Escala de Piers-Harris

\begin{tabular}{|lccccc|}
\hline \multicolumn{1}{|c}{ GRUPO } & $\mathrm{N}$ & $\overline{\mathrm{X}}$ & $\mathrm{DS}$ & Prueba $\mathrm{T}$ & \\
\hline $\begin{array}{l}\text { 1. Niños } \\
\text { Institucionalizados }\end{array}$ & 35 & 46.54 & 8.879 & $\mathrm{~T}=3.246$ & $\mathrm{G} 1=\mathrm{G} 2$ \\
$\begin{array}{l}\text { 2. Niños de zona } \\
\text { Marginal }\end{array}$ & 30 & 43.5 & 8.815 & & \\
$\begin{array}{l}\text { 1. Niños } \\
\text { Institucionalizados }\end{array}$ & 35 & 46.54 & 8.879 & $\mathrm{~T}=3.946^{*}$ & $\mathrm{G} 1>\mathrm{G} 2$ \\
$\begin{array}{l}\text { 2. Niños con } \\
\text { Atención } \\
\text { Psiquiátrica }\end{array}$ & 20 & 36.55 & 9.622 & & \\
$\begin{array}{l}\text { 1. Niños } \\
\text { de Zona Marginal }\end{array}$ & 30 & 43.5 & 8.815 & $\mathrm{~T}=2.665 *$ & $\mathrm{G} 1>\mathrm{G} 2$ \\
$\begin{array}{l}\text { 2. Niños con } \\
\text { Atención } \\
\text { Psiquiátrica }\end{array}$ & 20 & 36.55 & 9.622 & & \\
\hline
\end{tabular}

${ }^{*} \mathrm{p}<.05$.

Los puntajes alcanzados en autoconcepto por los grupos examinados seńalan en el Análisis de Varianza la existencia de diferencias entre los mismos $(\mathrm{kw}=14.20, \mathrm{~F}=7.827, \mathrm{gl}=2, \mathrm{p}=0.001)$. Estudiando con mayor precisión el comportamiento de esta variable se procedió al análisis intergrupal de las medias obtenidas (Prueba T). Los datos del Cuadro 2 
confirman que: a) No existen diferencias significativas entre los niños institucionalizados y los niños de zona marginal, ubicándose los mismos en un nivel promedio de autoconcepto; b) Como se esperaba se halló un bajo nivel de autoconcepto en el grupo de niños con atención psiquiátrica por lo que se establecen diferencias significativas en relación a este grupo, respondiéndose a nuestra interrogante.

Así mismo la Prueba $T$ confirma la existencia de diferencias significativas entre los niños diagnosticados como deprimidos de población clínico psiquiátrica y los niños no deprimidos, en cada uno de los factores y nivel general de autoconcepto de la Escala de Piers-Harris (DEP, $\mathrm{X}=36.5$, DS = 9.38; No DEP, $X=45.14, D S=8.84)$, siendo menor el nivel hallado en el primer grupo.

La validez de los instrumentos empleados y la representatividad de los grupos estudiados se confirman al haberse obtenido diferencias significativas entre ambos grupos de niños en relación a depresión, con la presencia de mayores puntajes en el CDI obtenidos por los niños de población clínica psiquiátrica (DEP, $\overline{\mathrm{X}}=16.1$, DS $=6.52$; No DEP, $\overline{\mathrm{X}}=12.76, \mathrm{DS}=6.37$ ).

Adicionalmente se obtuvieron datos importantes como, por ejemplo, la no existencia de una correlación significativa para la muestra total (n $=85)$ entre el nivel de depresión en el CDI y edad del niño $(r=0.0042$, $\mathrm{p}<0.05$ ), como también una baja correlación entre el nivel de autoconcepto y la misma variable, tanto a nivel general como en cada factor $(r$ $=-0.120, \mathrm{p}<0.05$ ).

Estudios sistemáticos con muestras más amplias podrán en un futuro examinar con mayor exactitud estos resultados. Sin embargo creemos que las distorsiones de tipo afectivo y cognitivo encontrados en la muestra de nińos estudiados, que interfieren en un normal desarrollo psicológico pueden explicar la relación entre estas variables, ya que la presencia del factor edad mantiene aún la relación entre mayor depresión y bajo nivel de autoconcepto en el niño. 


\section{Conclusiones}

1. La depresión en el niño es una variable que tiene relación con un bajo nivel de autoconcepto.

2. La variable institucionalización tiene mediana relevancia en el grado de depresión hallado en el niño interno, en cambio la variable socioeconómica si tiene relevancia en el grado de depresión manifestado por los niños examinados.

3. Las variables institucionalización del niño y nivel socioeconómico bajo no tienen relevancia en el nivel de autoconcepto hallado en los nińos examinados.

4. Los niños con diagnóstico clínico de depresión presentan mayores puntajes en el CDI y menores puntajes de autoconcepto en la escala de Piers-Harris.

5. El CDI y la escala de Piers-Harris son inventarios con propiedades adecuadas de validez y confiabilidad para medir la depresión y el desarrollo del autoconcepto en niños del medio peruano. En relación al CDI, se han encontrado características en las respuestas dadas al Inventario que permiten vincular la depresión en el nifio con problemas de tipo afectivo, relaciones interpersonales inadecuadas, imagen negativa de sí mismo y sentimiento de culpa e irritabilidad, datos importantes en el campo clínico para el diagnóstico y tratamiento de este desorden.

A partir de estos resultados hacemos las siguientes sugerencias:

1. Ejecutar acciones inmediatas a nivel de los organismos de Salud Mental del país en la prevención, diagnóstico y tratamiento de diversos desórdenes psicológicos en los sectores pobres y marginales, con especial atención en la niñez.

2. Investigar de forma amplia y sistemática la depresión en niños de nuestro medio, su prevalencia, causas, características, modalidades y aspectos de tratamiento, examinando su relación con otras variables vinculadas al desarrollo psicológico del nińo peruano. 
3. Realizar mayores estudios con el CDI, atendiendo a la confrabilidad test-retest, validez discriminativa en grupos de niños con diferente diagnóstico clínico y a su grado de correlación con otras variables psicológicas.

4. Diversificar el tratamiento clínico del niño deprimido considerando los aspectos cognitivo, afectivo y vegetativo, orientando la atención terapéutica a las siguientes áreas psicológicas: adaptación e integración social, vínculo afectivo, imagen personal y control de ideas e impulsos autodestructivos (culpa, irritabilidad, minusvalía).

5. Reestructurar los planes y acciones de la atención y tratamiento de los menores institucionalizados a partir de un enfoque más asistencial y específico y menos institucional y general, aspecto último que actualmente no contribuye a un adecuado desarrollo psicológico de estos nińos.

\section{Referencias}

Ajurriaguerra, J. de (1973). Manual de Psiquiatria Infantil. Barcelona: TorayMasson.

Alarcón R. (1986). Psicologia, Pobreza y Subdesarrollo. Lima: INIDE. American Psychiatric Association. (1980). Diagnostic and statistical manual of mental disorders (3rd ed.) Washington, DC: American Psychiatric Association.

American Psychiatric Association. (1988). Diagnostic and statistical manual of mental disorders (3rd ed. rev.) Washington, DC: American Psychiatric Association.

Avalos M. (1985). Estudio Comparativo entre el nivel de Inteligencia, Autoconcepto y Frustración en un grupo de niños. Lima: Tesis de Bachiller, Pontificia Universidad Católica del Perú.

Bell-Doland D., Reaven, N. y Paterson L. (1993) A Depression and Social Functioning: A Multidimensional Study of the Linkages. Journal of Clinical Child Psychology, 22, 306-315.

Bleschman E., McEnroe M. y Carella E. (1986). Chilhood Competence and Depression. Journal of Abnormal Psychology, 95, 223-227. 
Carey M. (1987). Children's Depression Inventory: Construct and

Discriminant Validity. Journal of Consulting and Clinical Psychology. 55, 755-761.

Castillo Rios, C. (1975). Los niños del Perú. Lima: Edit. Universo.

Cobos C. (1992) La Depresión Infantil. Madrid. Editorial Temas de Hoy. Finch, S., Saylor, C. y Edwards, G. (1985). Children Depression Inventory:

Sex and grade norms for normal children. Journal of consulting and clinical Psychology, 53, 424-425.

Frias, D., Del Barrio V., Garcia-Ross R. y Mestre V. (1992). Estructura familiar y Depresión Infantil. Anuario de Psicología. Universidad de Barcelona, 52, 121-131.

Green, B.J. (1980) Depression in early adolescence: An exploratory investigation of its frequency, intensity and correlates. Doctoral dissertation, Pennsylvania State University. Dissertation Abstracts International, 41, 3890-3891.

Grinspoon, L. (1982). Psychiatry, Anual Review. Washinton D.C: American Psychiatric. Press.

Helsel W.J y Matson J.L. (1984) The Assessment of depression in children: the internal structure of the child depression inventory (CDI). Behavorial Research and Therapy. 22, 289-298.

Hodges, K.K., Siegel, L., Mullins, L. y Griffin, N. (1983). Factor analysis of the children Depression Inventory. Psychological Reports, 53, 759763.

Kovacs, M. (1983). The Childrens' Depression Inventory. A Self Rated Depression scale for school - aged youngsters. University of Pittsburg School of Medicine. Material no publicado.

Lefkowitz, M y Burton N. (1978) Childhood depression: A critique of the concept. Psychological Bulletin. 85, 716-726.

Lefkowitz, M. y Tesiny E. (1980). Assessment of Chilhood Depression. Journal of consulting and clinical Psychology. 48, 43-50.

Lefkowitz, M. y Tesiny E. (1984). Rejection and Depression: Prospective and Contemporaneous Analyses. Developmental Psychology, 20, 776785 .

Lefkowitz, M. y Tesiny E. (1985). Depression in Children: Prevalence and correlates. Journal of consulting and clinical Psychology, 53, 647-656. Leintenberg, H., Leonard, W. y Carroll-Wilson M. (1986) Negative 
cognitive errors in children. Questionnaire development normative data, and comparisons betweeen children with and without self-reported symptoms of depression, low self-esteem, and evaluation anxiety. Journal of consulting and clinical psychology. 54, 528-536.

Malmquist, C.P. (1983) Major depression in Childhood: Why don't we know more? American Journal of Orthopsychiatry. 53, 262-268.

Puyuelo R. (1984). La Ansiedad Infantil: Un Dificil Bienestar. Barcelona. Herber.

Raffo, L.F. (1991). Depresión y Autoconcepto en niños Institucionalizados y no Institucionalizados. Lima: Tesis de Bachiller, Pontificia Universidad Católica del Perú.

Raffo, L.F. (1994). El Autoconcepto del Niño Escolar. Lima, PEA Asesores. Rueda, L. (1987). Relación entre autoconcepto y popularidad en un grupo de niños institucionalizados y no institucionalizados. Tesis de Bachiller, Pontificia Universidad Católica del Perú.

Saylor, C.F., Finch, A.J., Baskin, C.H., Furey, W. y Kelly M.M. (1984). Construct validity for measured of childhood Depression: Aplication of multitrait-multimethod methodology. Journal of consulting and clinical Psychology, 52, 977-985.

Stevenson, D. y Rommey D. (1984). Depression in Learning Disabled Children. Journal of learning disabilities. 17, 579-582.

Tems, C., Stewart, S., Skinner, J. y Hudhes, W. (1993). Cognitive Distortions in Depressed Children and Adolescents: Are They State Dependent or Traitlike? Journal of Clinical Child Psychology. 22, 316-326.

Tesiny, E. y Lefkowitz, M. (1982). Childhood Depression: A 6 month follow - up study. Journal of consulting and clinical Psychology, 50, 778780 . 